\title{
Individualised pulse oximetry limits in neonatal intensive care
}

R Gupta, C W Yoxall, N Subedhar, N J Shaw

\begin{abstract}
Aim-To determine whether individualised limits for arterial oxyhaemaglobin saturation by pulse oximetry $\left(\mathrm{SpO}_{2}\right)$ are more effective for detecting hypoxia and hyperoxia in sick newborn infants than setting fixed limits.

Methods-Six hundred and ninety two simultaneous measurements of $\mathrm{SpO}_{2}$ and partial pressure of oxygen in arterial blood $\left(\mathrm{PaO}_{2}\right)$ were made in 95 infants. The sensitivity and specificity for predicting hypoxia and hyperoxia using various fixed $\mathrm{SpO}_{2}$ limits and also individualised $\mathrm{SpO}_{2}$ limits, calculated using a standard equation, were determined and compared.

Results-None of the fixed limits for $\mathrm{SpO}_{2}$ was both sensitive and specific for predicting hypoxia and/or hyperoxia. There was no difference between these and individualised limits.

Conclusion-Individualised $\mathrm{SpO}_{2}$ limits are no more effective than fixed $\mathrm{SpO}_{2}$ limits for predicting hypoxia and/or hyperoxia in sick newborn infants. $\mathrm{SpO}_{2}$ monitoring is not an ideal method for assessing $\mathrm{PaO}_{2}$.
\end{abstract}

(Arch Dis Child Fetal Neonatal Ed 1999;81:F194-F196)

Keywords: pulse oximetry; intensive care; hypoxia; hyperoxia

The partial pressure of oxygen in the arterial blood $\left(\mathrm{PaO}_{2}\right)$ is the gold standard measurement of oxygenation in sick babies. However, pulse oximetry, to prevent chronic hypoxia and hyperoxia, is widely used to monitor arterial oxygenation in neonatal intensive care units. ${ }^{12}$ The position of the oxygen-haemoglobin dissociation curve (OHDC) is influenced by several factors - for example, temperature, $\mathrm{pH}$, percentage haemoglobin $\mathrm{F}$ and partial pressure of carbon dioxide in the arterial blood $\left(\mathrm{PaCO}_{2}\right) .^{3}$ The curve may therefore alter during the course of an infant's illness, making the estimation of arterial oxyhaemoglobin saturation using pulse oximetry $\left(\mathrm{SpO}_{2}\right)$ a poor predictor of $\mathrm{PaO}_{2}$. This, together with the sigmoid shape of the OHDC, makes it difficult to set acceptable upper and lower limits for $\mathrm{SpO}_{2}$. If the shape of an individual infant's OHDC could be determined by the most recent arterial blood gas result, thus taking into account the aforementioned influential factors, it might be possible to "customise" limits for an individual baby at any time by measuring $\mathrm{SpO}_{2}$ and $\mathrm{PaO}_{2}$ simultaneously, using a standard equation for the shape of the OHDC to predict which $\mathrm{SpO}_{2}$ levels correspond to the desired $\mathrm{PaO}_{2}$ range.

This study aimed to determine whether such individualised limits are more effective at detecting hypoxia and hyperoxia in sick babies than setting fixed limits.

\section{Methods}

Infants admitted to the neonatal intensive care units at the Liverpool Maternity Hospital and the Liverpool Women's Hospital, and who required arterial lines, were eligible for the study. Over three years (July 1994 to August 1997) simultaneous measurements of $\mathrm{SpO}_{2}$ and $\mathrm{PaO}_{2}$ were prospectively made by recording the infant's name and the $\mathrm{SpO}_{2}$ reading (from the Ohmeda oximeter being used on the infant for clinical purposes) on the blood gas result printout, when an arterial blood sample was taken for blood gas analysis. The $\mathrm{SpO}_{2}$ reading had to be stable for at least 30 seconds before the arterial blood sample could be taken, thus making it highly unlikely that the infant's $\mathrm{SpO}_{2}$ would be on the steep part of the OHDC (suggesting clinically significant hypoxia) at the time of sampling. As this was a pragmatic study, the results of which would hopefully be clinically applicable, no infant with hypotension was excluded. However, infants with structural congenital cardiac lesions were not studied. The date and time of blood sample analysis was automatically printed by the blood gas analyser. All blood gas printouts were stored for later analysis.

For each baby at least two sets of measurements were required, taken a maximum of 6 hours apart. For each pair of measurements the sensitivity and specificity of fixed $\mathrm{SpO}_{2}$ limits for predicting hypoxia and hyperoxia were calculated. Individualised $\mathrm{SpO}_{2}$ limits were then calculated retrospectively using a standard equation for the shape of the OHDC, devised by Tien and Gabel. ${ }^{4}$ These were then applied to the next pair of measurements in that individual. The sensitivity and specificity of the individualised limits for detecting hypoxia and hyperoxia were calculated and compared with those when using fixed limits.

Hypoxia was defined as a $\mathrm{PaO}_{2}$ of less than 6 $\mathrm{kPa}$ and hyperoxia as a $\mathrm{PaO}_{2}$ of more than 10 $\mathrm{kPa}$, using the recommendations of the Joint Working Group of the British Association of Perinatal Medicine and the Research Unit of the Royal College of Physicians. ${ }^{5}$

Results

During the study period 692 simultaneous measurements of $\mathrm{SpO}_{2}$ and $\mathrm{PaO}_{2}$ were made in 95 infants. The median (range) gestation was 
Table 1 Hypoxia prediction

\begin{tabular}{lll}
\hline Lower limit for $\mathrm{SpO}_{2}(\%)$ & Sensitivity & Specificity \\
\hline 85 & 0.11 & 0.90 \\
86 & 0.16 & 0.81 \\
87 & 0.23 & 0.83 \\
88 & 0.33 & 0.77 \\
89 & 0.41 & 0.67 \\
90 & 0.55 & 0.65 \\
91 & 0.72 & 0.56 \\
92 & 0.80 & 0.49 \\
93 & 0.84 & 0.39 \\
94 & 0.89 & 0.33 \\
95 & 0.94 & 0.27 \\
Individualised & 0.68 & 0.46 \\
\hline
\end{tabular}

Table 2 Hyperoxiaprediction

\begin{tabular}{lll}
\hline Upper limit for $\mathrm{SpO}_{2}(\%)$ & Sensitivity & Specificity \\
\hline 90 & 1.00 & 0.14 \\
91 & 1.00 & 0.15 \\
92 & 1.00 & 0.17 \\
93 & 1.00 & 0.20 \\
94 & 1.00 & 0.27 \\
95 & 1.00 & 0.40 \\
96 & 0.80 & 0.59 \\
97 & 0.42 & 0.92 \\
98 & 0.11 & 0.86 \\
99 & 0.06 & 0.75 \\
Individualised & 0.75 & 0.64 \\
\hline
\end{tabular}

28 (23 to 40 ) weeks and the median (range) birthweight was 1165 (590 to 3570) g. Thirty girls and 65 boys were studied. The sensitivities and specificities for hypoxia and hyperoxia for fixed and individualised limits for $\mathrm{SpO}_{2}$ are shown in tables 1 and 2 .

\section{Discussion}

To prevent potential morbidity in preterm infants, associated with episodes of chronic hypoxia and/or hyperoxia while ventilated, we studied the effect of using individualised $\mathrm{SpO}_{2}$ limits, but were unable to show any benefit compared with the use of fixed $\mathrm{SpO}_{2}$ limits.

Theoretically, because of the variable relation between $\mathrm{PaO}_{2}$ and arterial oxyhaemoglobin saturation $\left(\mathrm{SaO}_{2}\right)$ in sick infants, recalculating the shape of the OHDC in each infant after a blood gas analysis result should take into account some of the factors which alter its shape, and therefore provide $\mathrm{SpO}_{2}$ limits which are more appropriate for that infant. This should result in a more effective method to prevent hypoxia and hyperoxia in these patients. Unfortunately, in clinical practice, setting individualised limits, using the method described, was no more effective at predicting hypoxia than a lower fixed limit of $91 \%$, and it was no better at predicting hyperoxia than an upper fixed limit of $96 \%$.

There are several possible reasons for this. Setting individualised limits requires accurate simultaneous measurements of both $\mathrm{PaO}_{2}$ and $\mathrm{SpO}_{2}$. In clinical practice, on a busy neonatal unit, some of the measurements will probably not be accurate. Imprecisions in $\mathrm{PaO}_{2}$ measurements using standard blood gas analysers exist, ${ }^{6}$ and these may be greater on a busy neonatal unit when techniques for sampling and processing may not always be optimal. The $\mathrm{SpO}_{2}$ values produced correspond to real $\mathrm{SaO}_{2}$ values within about $\pm 2 \%$ and pulse oximetry is notoriously prone to artefact. ${ }^{7}$ Furthermore, the equation used to describe the shape of the OHDC may be inaccurate. However, of three equations for calculating $\mathrm{PaO}_{2}$ from $\mathrm{SaO}_{2}$, assessed by Gabel, the equation produced by Tien and Gabel was found to be most accurate. ${ }^{8}$ Finally, the position of the OHDC may move frequently in an individual infant. Some of the infants in the study would have received red cell transfusions between blood gas measurements and this may have affected the position of the OHDC by altering the amount of circulating haemoglobin F. Although this theoretically affects the OHDC, it has not been shown to have a major effect clinically, ${ }^{9}$ and is unlikely to have affected our results.

On a practical level, individualising $\mathrm{SpO}_{2}$ limits has its difficulties. Firstly, calculating individualised limits is complex. Secondly, the calculated limits would have to be communicated to the member of nursing staff looking after the infant on each occasion, and care would have to be taken to ensure that the limits were communicated at each nursing and medical handover. Thirdly, continuously changing limits may lead to confusion.

Individualised $\mathrm{SpO}_{2}$ limits could, however, be used on an ad hoc basis if there is a trend towards too high or too low $\mathrm{PaO}_{2}$ readings compared with the monitored $\mathrm{SpO}_{2}$ readings in a particular infant. This should be reviewed frequently especially if the clinical state of the infant changes.

Oxygen saturation monitors were not designed specifically for preventing hypoxia and hyperoxia. A better way of non-invasively assessing $\mathrm{PaO}_{2}$ than saturation monitoring is transcutaneous oxygen monitoring. However, many units use saturation monitors to monitor oxygenation and the sensitivities and specificities in tables 1 and 2 can be used to help determine the most appropriate limits for $\mathrm{SpO}_{2}$. Our results suggest that the best fixed lower and upper limits for $\mathrm{SpO}_{2}$ are $91 \%$ (sensitivity of 0.72 and specificity of 0.56 ) and $96 \%$ (sensitivity of 0.80 and specificity of 0.59), respectively, but these should be used only as a guide. Previous authors have suggested lower limits for $\mathrm{SpO}_{2}$ ranging from 89 to $93 \%,{ }^{310}$ and upper limits for $\mathrm{SpO}_{2}$ ranging from 95 to $97 \%$. $^{3-11}$

In summary, when using $\mathrm{SpO}_{2}$ monitoring to assess oxygenation, individualised $\mathrm{SpO}_{2}$ limits are no better than fixed limits for preventing hypoxia or hyperoxia.

1 Willis N, Mogridge J. Indicators of hypoxia. Acta Anaesthesiol Scand (Suppl) 1995;107:45-8.

2 Flynn JT, Bancalari E, Snyder ES. A cohort study of transcutaneous oxygen tension and the incidence and severity of retinopathy of prematurity. $N$ Engl $\mathcal{F}$ Med 1992;326:1050-80.

3 Hay WW Jr, Thiloe E, Curlander JB. Pulse oximetry in neonatal medicine. Clin Perinatol 1991;18:441-71.

4 Tien YK, Gabel RA. Prediction of $\mathrm{PO}_{2}$ from $\mathrm{SO}_{2}$ using the standard oxygen haemoglobin equilibrium curve. $\mathcal{F} A p p l$ Physiol 1977;42:985-7.

5 Anonymous. Development of audit measures and guidelines for good practice in the management of neonatal Group of the British Association of Perinatal Medicine Group of the British Association of Perinatal Medicine Arch Dis Child 1992;67:1221-7.

6 Radiometer Ltd. ABL 520. User's Handbook (version 13.0). Crawley: Radiometer, 1993. 
7 Fanconi S, Tschupp A. Accuracy of a new transmittancereflectance pulse oximetry sensor in critically ill neonates. 8 Crit Care Med 1994;22:1142-6.

and acid-base variables. Respir Physiol 1980;42:211-32. and acid-base variables. Respir Physiol 1980;42:211-32.
Pologe JA, Raley DM. Effects of fetal hemoglobin on puls oximetry. $\mathcal{F}$ Perinatol 1987; 7:324-6.
10 Hay WW Jr, Brockway JM, Eyzaguirre M. Neonatal pulse oximetry: accuracy and reliability. Pediatrics 1989;83:71722.

11 Poets CF, Wilken M, Seidenberg J, Southall DP, von der Hardt H. Reliability of a pulse oximeter in the detection of hyperoxaemia. F Pediatr 1993;122:87-90. 